\title{
APROXIMACIÓN AL PROCESO PRODUCTIVO DEL SISTEMA SANITARIO ESPAÑOL
}

\author{
Autoras: Sophie Gorgemans \\ Departamento de Dirección y Organización de Empresas \\ Universidad de Zaragoza \\ Olga Urbina ${ }^{1}$ \\ Departamento de Dirección y Organización de Empresas \\ Universidad de Zaragoza
}

\section{Resumen}

En este trabajo se aborda el análisis del proceso de producción en el ámbito sanitario, una cuestión de gran relevancia para avanzar en la utilización de técnicas que evalúen el funcionamiento de los servicios sanitarios y poder, así, tomar decisiones que permitan continuar su proceso de mejora. Se presenta una reflexión sobre las principales dificultades para delimitar y medir el producto sanitario, centrando el análisis en que sea útil para analizar la gestión de los centros sanitarios. El propósito del trabajo es estudiar todas aquellas dimensiones que

\footnotetext{
${ }^{1}$ ourbina@ unizar.es
} 
permitan acercarnos a una medida del producto viable, a través de una integración de sistemas de clasificación de pacientes y medidas de la calidad técnica de los servicios asistenciales que se prestan en el sistema sanitario.

Palabras clave: producción sanitaria; atención primaria; hospitales; medida del producto; sistema de ajustes por riesgo; calidad.

\title{
Production Process in the Health System
}

\begin{abstract}
In this paper, we analyse the production process in the health sector, an important matter to advance in the use of techniques to assess the health services management as well as to take decisions in order to maintain the improvement. We make a specific reflection on the main difficulties to mark off and measure the health product, focusing the analysis on a measure of product which is useful for evaluating and comparing how health centers are managed. The purpose of this paper is to address all dimensions that allow us to approach a measure of viable product through the integration of patient risk classification and technical quality measures of health care services provided in the health system.
\end{abstract}

Key words: health production; primary care; hospitals; product measure; adjust risk patient classification; quality.

\section{INTRODUCCIÓN}

En estos momentos el déficit público y las dificultades económicas son algunos de los principales problemas por los que atraviesa nuestro país. Ante esta situación mantener el estado de bienestar es uno de los temas redundantes en las discusiones de los gobernantes. Para conseguirlo es necesario controlar la expansión del gasto público sin mermar la calidad de los servicios ofrecidos, para lo que es imprescindible contar con información que permita analizar la forma de prestar los servicios públicos y que sirva para tomar decisiones con las que se consigan estos propósitos.

Uno de los servicios claves del estado de bienestar es el sanitario, en este ámbito se han ido introduciendo reformas con las que se pretende conseguir mejorar 
los servicios y contener el aumento del gasto. Algunas de las reformas más significativas son las que se señalan a continuación.

La Ley General de Sanidad de 1986 es la primera ley que considera la sanidad en su conjunto y que define el concepto de Sistema Nacional de Salud, integrado por el conjunto de los servicios regionales de salud de las comunidades autónomas. Cada una de las 17 comunidades se encarga de establecer la estructura de los servicios de salud, donde aparecen como elemento fundamental el área de salud $^{2}$. Los servicios ofrecidos por cada una de las áreas se agrupan en dos niveles asistenciales:

Atención primaria: constituye el primer nivel de asistencia y se convierte en la puerta de entrada del paciente al sistema sanitario. En los centros de salud, ubicados en las zonas básicas de salud, es donde se realizan los procesos sanitarios de atención primaria. Dentro de la cartera de servicios que se ofrece destaca el desarrollo de programas y funciones de promoción y prevención, curación y rehabilitación.

Atención especializada: es el segundo nivel de asistencia y se realiza en los hospitales y centros de especialidades, prestando servicios de mayor complejidad. En cada área de salud se dispone al menos de un hospital.

Durante los años 80 también comienza el proceso de transferencia de las competencias sanitarias a las comunidades autónomas, con el propósito de conseguir una mayor descentralización en las decisiones. Este proceso termina en 2002 con la transferencia de competencias en sanidad a las 10 comunidades autónomas que quedaban por asumirlas.

La pieza básica de las reformas, recogida en el Informe Abril (1991), ha sido la introducción de cierta descentralización en el sistema, mediante la separación entre las funciones de financiación, compra y provisión, propuestas que se encuentran en la misma línea que las de otros países de nuestro entorno (Saltman y Figueras, 1997; Saltman y Bankauskaite, 2006). Un reflejo de esta propuesta se encuentra en la Ley 15/1997 de 25 de abril, sobre habilitación de nuevas formas de gestión del Sistema Nacional de Salud, a partir de la cual se comienza a poner en marcha una amplia gama de formas y tipologías organizativas en el ámbito sanitario. Cada comunidad autónoma elige la forma de organizar su sistema sanitario creándose gran variedad de fórmulas para ofrecer los servicios sanitarios. En el trabajo realizado por López Casasnovas y otros (2005) se pone de relieve que, a pesar de estas diferencias, se sigue manteniendo la equidad en los servicios sanitarios que se prestan a los ciudadanos. Salud.

${ }^{2}$ El trabajo de García-Armesto y otros (2010) ofrece una visión detallada del Sistema Nacional de 
Como se puede ver el sistema sanitario se encuentra inmerso en cambios continuos y debe responder ante la sociedad a los principios de eficacia, eficiencia y calidad para obtener una mejora de la salud de la población. En este contexto, donde las necesidades presupuestarias en sanidad son ilimitadas y sin embargo ocurre lo contrario con los recursos, es obvio que la gestión de estos recursos es un tema de enorme interés para la sociedad. En este sentido, desde un planteamiento macroeconómico, no se pueden pasar por alto los costes de oportunidad que originan las decisiones sobre el reparto de recursos públicos entre las diferentes necesidades de un país o comunidad autónoma, lo que pone de manifiesto la necesidad de contar con toda la información necesaria que permita tomar las decisiones correctas.

Dada la limitación presupuestaria, se impone intentar obtener el máximo beneficio social de las inversiones. Para las unidades de gestión, esta limitación de los presupuestos implica la correcta planificación de las necesidades y el ajuste del gasto al dinero asignado, en el contexto de una progresiva integración de técnicas de gestión empresarial en el ámbito sanitario que permitan conseguir una mejora de la asistencia sanitaria. Las herramientas de gestión en el ámbito de meso y micro-gestión tratan de conseguir un mayor nivel de eficiencia, algunas de ellas quedan materializadas en la aplicación de la filosofía Lean que promueve la eficiencia, centrándose principalmente en el valor que se genera al paciente y eliminando todos los costes innecesarios (Crema y Verbano, 2015). El interés por esta herramienta es creciente y se han desarrollado algunas aplicaciones que permiten mejorar la productividad en el ámbito sanitario (D'Andreamatteo y otros, 2015). Además es necesario introducir la utilización de técnicas capaces de evaluar la eficiencia del funcionamiento de las organizaciones sanitarias, técnicas que permitan utilizar el benchmarking con el propósito de que los resultados obtenidos sirvan para mejorar la planificación y sirvan como referente para tomar decisiones de actuación que mejoren la gestión del sistema sanitario.

Las reformas, como se ha visto, se han dirigido hacia una mayor descentralización y una separación de funciones. Ante esta situación, es necesario contar con herramientas que permitan una evaluación precisa y una medida de la actividad asistencial realizada en el ámbito sanitario. Esta evaluación no está exenta de complicaciones ya que el sistema sanitario como productor de servicios se enfrenta a dificultades en la delimitación y medida de su producción.

En este trabajo se pretende abordar una aproximación al proceso de producción del sistema sanitario, diferenciando entre el primer y el segundo nivel. Se ponen de relieve los principales aspectos que se deben considerar en la medida del producto y se presentan las dificultades inherentes a la definición y utilización de outputs adecuados para analizar el funcionamiento de los centros sanitarios. Este análisis nos permite avanzar hacia una propuesta de conceptualización y medición del producto sanitario, en la que se consideran tanto la actividad sanitaria como el tipo de paciente que se atiende y, por supuesto, la calidad con la que se presta 
el servicio. Es clave considerar estas tres cuestiones para llegar a una medida más cercana a la realidad que permita a los centros corregir y mejorar su gestión, además de sus niveles de calidad asistencial. Para ello la metodología utilizada en este artículo se basa en una extensa revisión de la literatura. Esta revisión se ha llevado acabo utilizando las siguientes palabras clave en castellano y su traducción al inglés: producción sanitaria, atención primaria, hospitales, medida del producto, sistema de ajustes por riesgo, calidad, como términos de búsqueda en las principales bases de datos bibliográficos (Web of Knowledge, Scopus, Dialnet, ABI Inform).

\section{EL PRODUCTO SANITARIO}

La definición, delimitación y medida del producto sanitario constituye, para gestores e investigadores en Economía de la Salud, un punto de reflexión importante dado que sobre este concepto descansa buena parte de las valoraciones de productividad y de eficiencia que permiten evaluar las organizaciones sanitarias. La medida del output sanitario presenta dificultades ligadas a las características del servicio prestado, dificultades que son comunes a todas las organizaciones del sector servicios.

El panorama sobre medición del producto de los servicios públicos es decepcionante, se encuentra aún en sus primeros $\operatorname{pasos}^{3}$ y la situación no ha mejorado demasiado desde que, años atrás, Hill (1977) apuntaba: "es una triste reflexión sobre el Estado de la Economía que haya tan poca percepción sobre las características físicas de la mayoría de los servicios y los productos de industrias principales como la sanidad ${ }^{4}$ y la educación, y se midan usualmente por sus factores haciendo que las medidas sean inútiles para la mayor parte de los fines". A continuación se presenta un análisis de las dificultades de medición del producto ligadas a la naturaleza de los servicios y, por extensión, un análisis del producto sanitario.

El sector sanitario queda enmarcado dentro del sector servicios y en las organizaciones de este sector la delimitación del concepto y la medida del producto son aspectos complejos. El estudio genérico de las características de los servicios puede constituir un paso previo para el análisis de la producción en los servicios sanitarios, conocer sus características nos puede servir para poner de relieve las principales limitaciones en la definición del producto sanitario.

\subsection{Medida del producto en el sector servicios}

Las principales características de los servicios son: intangibilidad, simultaneidad en el proceso producción-consumo, intensa utilización de recursos humanos cuya

\footnotetext{
${ }^{3}$ Véase Domínguez y Rueda (2005).

${ }^{4}$ El sector sanitario forma parte según la clasificación de Kent (1985) de los servicios de consumo masivo.
} 
actitud resulta clave en la calidad final de la prestación, intervención directa del consumidor en el volumen de producción, fugacidad (los servicios no pueden ser ni almacenados, ni inventariados), el consumidor tiene acceso al servicio pero no puede apropiarse del mismo (Fletcher y Snee, 1985). De todas estas peculiaridades, las dos primeras implican una mayor dificultad para medir el producto. Su naturaleza intangible explica la dificultad que existe para diferenciar entre lo que es el proceso productivo y lo que es el propio producto. En este sector no se realiza una transacción de un producto observable directamente, del cual se pueden conocer de forma instantánea las características que tiene. Otra de las peculiaridades, es que los usuarios o clientes de estos servicios intervienen de forma directa en el momento de la producción. En muchos servicios, como sanidad, justicia y educación, el proceso de producción se origina en el momento que se produce la demanda del propio usuario, de forma que la producción está condicionada en gran medida por las características que estos presentan ${ }^{5}$. Otra dificultad en realizar la medida reside en el carácter multidimensional del producto de los servicios, en este sentido es posible que algunos de los elementos sean cuantificables mientras otros no (González y otros, 1989).

Las características de los servicios generan, por lo tanto, una serie de limitaciones que explican las dificultades que se encuentran en conseguir una medida del producto. Sin embargo, se han realizado esfuerzos, tanto por parte de los gestores como por parte de los investigadores, para cuantificar la producción. Así, en la literatura, han aparecido varias consideraciones con las que se pretende obtener una medida de la producción en el sector servicios (Kendrick, 1985; Ross y Burkhead, 1974 y Bradford y otros, 1969). En la Tabla 1 se resumen las distintas medidas del producto que se utilizan en el sector de los servicios.

\section{Tabla 1. Medidas de la producción en el sector servicios}

\begin{tabular}{|c|l|}
\hline Medidas & \multicolumn{1}{c|}{ Definición } \\
\hline Throughput & Se basa en considerar los recursos consumidos (inputs) \\
\hline Output & Utiliza como base de medida la actividad realizada \\
\hline Outcome & Se refiere a los resultados obtenidos con los servicios ofrecidos \\
\hline
\end{tabular}

Fuente: elaboración propia.

La primera medida del producto se conoce como Throughput y se define como el conjunto de materiales que se consumen durante el proceso. Se trata, por tanto, más de una aproximación a los inputs utilizados en la producción que al producto fabricado o servicio prestado. Esta medida ha sido poco utilizada y sustituida por otros métodos.

\footnotetext{
${ }^{5}$ Véase Griliches (1992).
} 
La segunda aproximación a la medida del producto del sector servicios, que se conoce como medida del Output, es la que toma como unidad de análisis el conjunto de actividades o servicios realizados por lo que se centra en los productos intermedios (Pallot, 1991 y McCulloch y Ball, 1992). En este caso, se supone que un mayor nivel de actividad contribuye a una mayor cantidad de producción. Esta aproximación se ha utilizado en diversos servicios públicos sobre todo en trabajos empíricos de análisis de eficiencia, entre los que se puede citar la educación (enseñanza universitaria y no universitaria), la justicia, los servicios de protección civil (bomberos), los de recogida de basura y, por supuesto, la sanidad.

La última propuesta de medida del producto se define a partir del Outcome que representa las consecuencias que los outputs han producido en la sociedad, es decir, el resultado obtenido con los servicios ofrecidos. Se trata del impacto que tienen las actividades llevadas a cabo por el gobierno en relación con las metas por lo que, en algunos servicios, como los de policía o de salud, puede resultar más fácil hacer una afirmación acerca del impacto de una medida política que acerca de su resultado. Efectivamente, en muchos casos, es difícil determinar cuál es exactamente el resultado, distinguiendo lo que se ofrece de los resultados alcanzados. Esta medida, en la mayoría de los casos, está influida por variables que escapan al control del productor, por lo que son medidas poco efectivas para utilizarlas en gestión.

Dado que las características del sector servicios son extensibles al sector sanitario también lo son las dificultades encontradas en la medida del producto. Todas estas características son las que permiten reflexionar sobre la dificultad de medir el producto sanitario. De hecho, Newhouse (1993:238) indica que "la magnitud de las ganancias de productividad en atención médica es una cuestión tremendamente difícil debido a la dificultad para medir el producto". A continuación nos vamos a centrar en definir el producto en el ámbito sanitario.

\subsection{El producto en el sector sanitario}

En la función de bienestar social, la variable salud tiene dos componentes: curar y cuidar (López, 2003:16) entendidos como valor de uso (efectividad o impacto de lo que se gasta) y valor de cambio (utilidad o posibilidades de acceder a ciertos bienes). Los resultados del servicio prestado por un sistema sanitario deberían valorarse, por tanto, en la medida en la que desembocan en mejoras en la salud asignándole un valor (monetario o no). En este sentido se expresa el informe mundial de la Salud (OMS, 2000: XIII): "la mejora de salud es, sin duda, el principal objetivo de un sistema de salud, pero no el único. En realidad, el objetivo de una mejor salud tiene dos vertientes: por un lado, se debe alcanzar el mejor nivel posible; por otro lado, se debe procurar que haya las menores diferencias posibles entre las personas y los grupos (o sea, el sistema debe ser equitativo)". 
Para este organismo mundial, un sistema de salud se considera bueno cuando el paciente recibe un diagnóstico correcto y los servicios terapéuticos le conducen al estado óptimo de salud que puede alcanzar, dado el estado de los conocimientos médicos y de la disposición de tecnologías (Rubilar y Rossi, 2005).

El impacto de los servicios sanitarios suele evaluarse en términos de ganancia en esperanza de vida o de reducción en la mortalidad a lo largo de los años. En realidad, el peso que pueden tener los servicios de salud sobre el estado de salud tiene un papel más parco frente a las mejoras en nutrición, higiene y manejo de la reproducción, como recogen Fogel (1994) y McKeown (1976). Otros estudios (Marmot y otros, 1994 y Wilkinson, 1996) han confirmado la importancia de las variables socio-económicas para explicar las diferencias en el estado de salud entre los individuos Se valora, por tanto, la aportación de los servicios sanitarios a la mejora de la salud de forma residual y se sitúan las causas de esta mejoría en los avances en salud pública, nivel socio-económico y educativo, y en los cambios en los estilos de vida. No obstante, existen componentes atribuibles a los servicios sanitarios cuya importancia irá creciendo a medida que nos adentramos en el siglo XXI porque los niveles de salubridad y bienestar son ya elevados. La transición tecnológica está aportando un compendio de herramientas novedosas en materia preventiva, terapéutica o de diagnóstico que permiten reducir el peso de la enfermedad. El impacto de los servicios sanitarios sobre la salud en entornos distintos puede ser muy diferente así como lo demuestran Ortún y otros (2004), en el caso del infarto agudo de miocardio en Estados Unidos y España. Estos autores llegan a la conclusión de que la contribución de los servicios sanitarios a la mejora de la salud de la población no debería asumirse con carácter general sino estudiarse en cada caso, es decir, para cada enfermedad concreta y cada país concreto.

En la producción de un sistema sanitario el paciente forma parte del proceso de producción, lo que dificulta enormemente que se pueda establecer una estandarización del producto ofrecido La interacción del cliente en el proceso de producción es lo que explica la heterogeneidad de los productos. En definitiva, las características del paciente varían la demanda de productos y, por tanto, el proceso de producción en cada caso. Si se lleva esta cuestión al extremo se podría decir que cada paciente recibe un servicio determinado y diferente del resto. Los pacientes contribuyen al proceso de producción, interactúan con los profesionales sanitarios para facilitarles información sobre su estado de salud y sus síntomas, de forma que, con esta información, puedan realizar un diagnóstico, es decir, que su integración en el proceso de producción es tal que, incluso podría hablarse de la co-producción de salud (Realpe y otros, 2015).

En base a la tipología de medidas de producto del servicio definidas en la tabla 1, se podría entender que el outcome sanitario es la mejora de la salud de la población. Cada individuo nace con un stock de capital "salud" que pierde valor a lo largo del tiempo y que se esfuerza en mantener a base de inyecciones 
de capital (inversiones en comportamientos saludables y consumos sanitarios) y en las atenciones sanitarias proporcionadas por los sistemas sanitarios dadas las condiciones del entorno socioeconómico en el que se encuentra dicho individuo (Rubio, 1998). Rognehaugh (1996) define outcome sanitario como el resultado de un proceso de prevención, detección o tratamiento, se trata de un indicador de la efectividad de las medidas sanitarias realizadas sobre los pacientes. Esta medida es limitada porque el resultado esperado expresado en términos de mejoras de salud está influido por variables externas (Grossman, 1979). La mejora de salud está relacionada con el estado de bienestar y la calidad de vida que gana el paciente y la perspectiva temporal de la misma Las técnicas de medida del outcome incluyen cuestiones relativas a la autopercepción de la salud e índices de utilidad que se basan en las preferencias o utilidades que dan los individuos a los diferentes estados de salud En este sentido, están adquiriendo una creciente importancia las mediciones de resultados de salud percibidos por el paciente ("Patients reported outcomes" o PRO de los anglosajones). Esta tendencia es, sin lugar a duda, un reflejo de una mayor consideración del paciente en el proceso sanitario.

La aproximación throughput al producto sanitario se puede realizar valorando los inputs que se utilizan para prestar el servicio sanitario (número de profesionales sanitarios, recursos técnicos, etc.). Según indica Peiró (1998:199), la medida de la productividad de los servicios sanitarios se enfrenta a problemas conceptuales de definición del producto pero también del recurso. La definición de los recursos debería hacerse en términos de unidades equivalentes de tiempo (horas de jornada laboral, camas instaladas, equipos de alta tecnología, etc.).

La definición habitual del output sanitario recoge la diferencia entre los productos finales y los productos intermedios (Martínez, 2007). Así, siguiendo la propuesta del Ministerio de Salud y Consumo (Gestión analítica, 1990) se pueden definir los productos finales como aquellos que dan respuesta a un problema de salud (por ejemplo tratamiento de un melanoma) y los productos intermedios como los que forman parte de un proceso asistencial y que requieren de una agrupación de recursos y conocimientos específicos (por ejemplo consulta médica, pruebas diagnósticas). Si lo que utilizamos es la definición de output, la medida del producto sanitario se puede realizar contabilizando los actos asistenciales brutos (consultas, altas, etc.) es decir las actividades o procesos que realizan los profesionales en las instituciones sanitarias directamente sobre el paciente y que permiten evitar las enfermedades o mejorar el estado de salud. Por tanto, podemos decir que la atención ofrecida al paciente, el diagnóstico y tratamiento ofrecido en los centros sanitarios, así como la forma de realizar la prestación de estos servicios (output) interfiere directamente sobre la mejora de salud que experimenta el paciente (outcome). En este proceso que lleva a esta mejora en la salud, un elemento importante que se debe valorar es el nivel de calidad técnica, una variable de gran relevancia en los procesos de producción sanitaria, que se explica en el presente trabajo. 
Como se ha observado a lo largo de estas líneas, la dificultad reside en definir el concepto de producto sanitario pero aún más si se quiere establecer una medida que permita realizar evaluaciones objetivas y comparativas en este sector. Estudiar la actividad de producción nos permite entender la forma de trabajar del sistema sanitario y la manera de obtener el resultado final sobre los pacientes.

\section{EL PROCESO DE PRODUCCIÓN EN SANIDAD}

El análisis del proceso de producción del sistema sanitario puede abordarse considerando que se trata de una organización cuyo principal objetivo es la creación de valor. Para conseguirlo necesita utilizar y combinar, con una tecnología determinada, una serie de factores productivos para obtener los productos necesarios que finalmente permitan llegar al objetivo propuesto de crear valor. En el sistema sanitario la creación de valor se traduce en una mejora de la salud de la población o un aumento de la calidad de vida del paciente.

Reflexionar acerca del proceso productivo es necesario para identificar con mayor acierto los inputs y outputs a utilizar en la evaluación del funcionamiento de estos servicios. El estudio de la producción sanitaria se puede realizar desde una perspectiva integral, considerando el sistema de salud en su conjunto o desde una perspectiva desagregada, analizando de forma individual cada uno de los niveles asistenciales. En el caso de tomar como ámbito de referencia el sistema de salud, se contempla el proceso de producción como la integración de todos los niveles asistenciales. Desde la perspectiva del resultado que busca el paciente cuando demanda estos servicios, éste puede obtenerse en el primer nivel asistencial o puede que sea necesario recurrir a la intervención de los especialistas, entrando en el segundo nivel asistencial. En cualquier caso, esta decisión queda a discreción del profesional sanitario de atención primaria, cuando el funcionamiento del sistema se realiza con normalidad Ante esta perspectiva, cabe destacar la importancia que tiene la integración de los dos niveles asistenciales para conseguir un funcionamiento adecuado del sistema sanitario (Gimeno y otros, 2006). El estudio de la producción sanitaria en la que se consideran los dos niveles asistenciales permite establecer un análisis desde una perspectiva global e integradora. Aunque realmente la población de pacientes que se trata es diferente y requiere de procesos de producción distintos. Por tanto, la organización del sistema sanitario en dos niveles asistenciales diferentes y la complejidad de los mismos justifican el desarrollo de un análisis separado por niveles como lo presentamos a continuación.

En ambos niveles sanitarios el paciente juega un papel relevante en los procesos de producción, aunque pierda en gran medida sus posibilidades de tomar decisiones y no pueda valorar, a corto plazo, la calidad del servicio prestado. El profesional sanitario se apodera de las decisiones de éste y genera la demanda y oferta de servicios médicos/quirúrgicos y tratamientos/cuidados. 


\subsection{Proceso de producción en atención primaria}

En los países en los que sus sistemas de salud se centran en la atención primaria se consigue unos mejores niveles de salud y una mayor satisfacción de la población de sus servicios sanitarios y una mejor redistribución de los recursos En nuestro país la atención primaria funciona como el nivel de entrada de los pacientes en el sistema sanitario (gatekeeper), algunas de sus principales características, tal como recogen Zurro y Jodar (2011), son la de accesibilidad (para facilitar la utilización de los servicios a la población que lo necesite), continuidad y longitudinalidad (basadas en la relación a lo largo del tiempo y la coordinación de las actuaciones asistenciales) e integralidad (no solo se centra en la curación sino en la promoción de la salud y prevención de riesgos para la población). Los centros de salud a través de los equipos de atención primaria son los encargados de desarrollar funciones de docencia dirigidas a promocionar y prevenir riesgos de la salud, de gestión para planificar los recursos, de investigación y fundamentalmente están destinados a cubrir las necesidades de salud de la población (mantenerse sano, recuperar la salud, vivir con la enfermedad y llegar al final de la vida en condiciones dignas) (De los Santos Ichaso y Garcia-Armesto, 2012).

En este apartado se trata de abordar desde la perspectiva de la asistencia sanitaria la forma de desarrollar en atención primaria este proceso de producción. En cualquier proceso de producción se estudia la relación que existe entre los recursos utilizados para la producción y los productos obtenidos. Sin embargo, el estudio del proceso de producción en sanidad es mucho más complejo debido a que, por un lado, el producto se encuentra muy influido por variables externas al propio proceso de producción y, por otro lado, en sanidad el paciente valora tanto el producto que recibe como el propio proceso de producción.

En la Figura 1 se representa de forma resumida el proceso de producción en atención primaria. En la parte izquierda de la figura, se refleja el inicio del proceso de producción, el cual se origina en el momento en el que el paciente solicita los servicios sanitarios o entra en el centro de salud En este nivel asistencial, y como elemento diferencial, se tienen que considerar las actividades preventivas, en este caso, es el propio sistema sanitario el que llama al paciente para comenzar el proceso de producción. Para iniciar el proceso juega un papel importante los servicios de citación de los pacientes y de información de los mismos, que se pueden considerar como procesos de apoyo a la producción de servicios sanitarios. Por otro lado, como complemento a los servicios sanitarios dentro del centro de salud se encuentran los servicios sociales que ofrecen principalmente servicios de tipo social y económico y facilitan los servicios dirigidos a la población con dependencia, principalmente a las personas mayores. Se trata por tanto, de servicios que pueden considerarse un apoyo a los servicios sanitarios y con los que, en muchas ocasiones, deben trabajar de forma conjunta. 
Una vez iniciado el proceso de producción sanitaria, se pueden diferenciar en él dos niveles productivos. En el primero se producen los outputs o productos intermedios, en este nivel se combinan una serie de recursos o factores productivos con los que se consiguen los servicios asistenciales, se consideran todos aquellos recursos humanos y técnicos necesarios para realizar el proceso productivo primario y ofrecer a los pacientes los servicios asistenciales necesarios.

\section{Figura 1. Proceso de Producción en Atención Primaria}

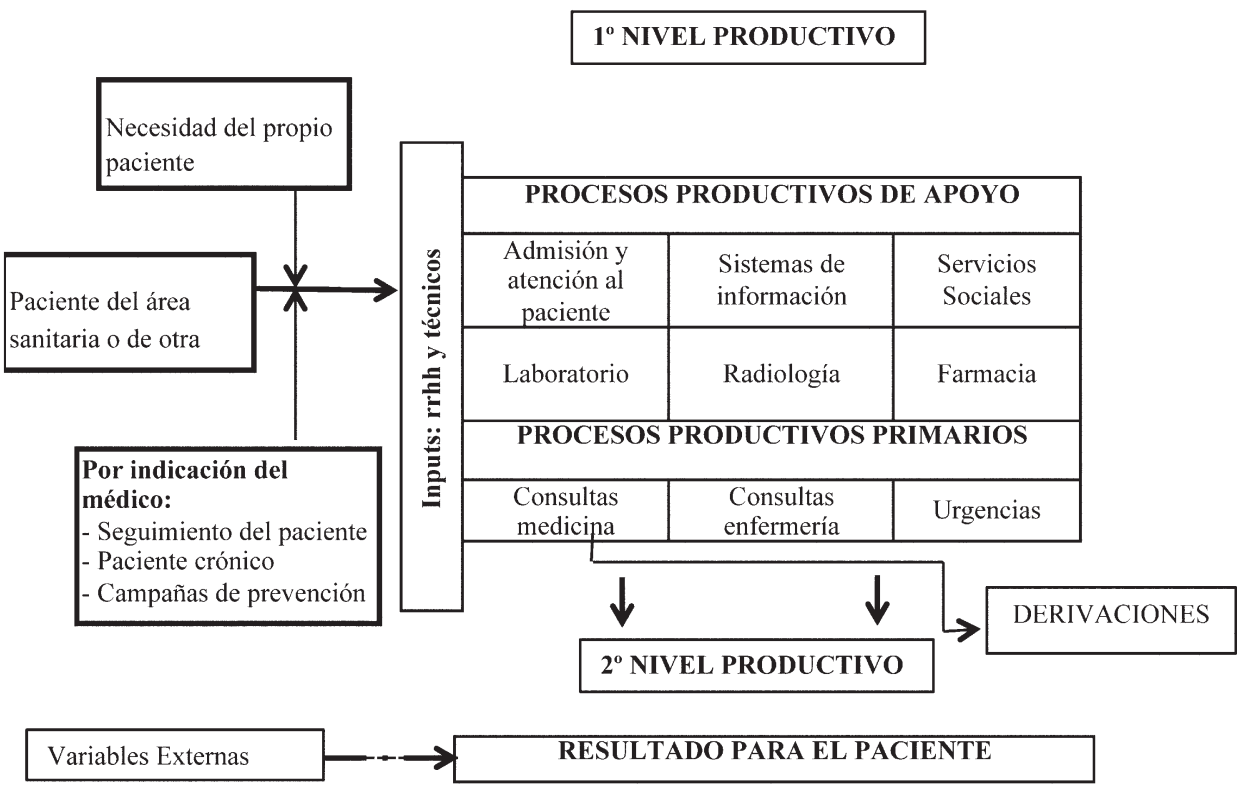

Fuente: elaboración propia a partir de Urbina (2002).

Dentro de este primer nivel productivo se lleva a cabo un proceso clínico de diagnóstico y tratamiento, donde el paciente, y la información que éste proporciona al profesional sanitario, juega un papel de gran importancia, en ocasiones para conseguir aumentar la información que permita al profesional realizar el diagnóstico correcto se solicitan pruebas complementarias como: análisis, radiografías etc. También es en este primer nivel de producción donde se valora si el problema del paciente se puede resolver en el propio centro de salud o si es necesario que se produzca una derivación del paciente hacia la asistencia especializada. En este primer nivel productivo se producen los productos intermedios, materializados a través de las consultas que se realizan por los diferentes profesionales sanitarios (medicina y enfermería). Las consultas se originan a demanda del propio paciente, para que se 
les atienda en el centro de salud o en el domicilio (ya sea con cita previa o a través de urgencias) o pueden ser concertadas por el propio profesional para realizar un seguimiento del paciente, sobre todo cuando se trata de enfermedades crónicas.

En el segundo nivel productivo los productos intermedios se convierten en los inputs necesarios para obtener resultados sobre la salud de la población atendida. El resultado (outcome) conseguido en este nivel se entiende, en un sentido amplio, como el cambio en el estado de salud de los pacientes o la mejora en la calidad de vida del paciente.

Al contemplar el proceso en su globalidad, se observa que la utilización de unos recursos humanos y técnicos debe revertir en una mejora en la salud de los pacientes o en una mejora en la calidad de vida del paciente. Sin embargo, en este proceso de producción el resultado que se consigue no es atribuible en su totalidad a las actividades desarrolladas en el centro de salud, ya que existen variables externas al centro que influyen en los resultados obtenidos en el proceso de producción, tal y como recoge Grossman (1979). De forma que, el resultado conseguido sobre la salud del paciente no depende únicamente de la efectividad del proceso de producción, depende también de factores del entorno, de características que recogen el estilo de vida de la población y los factores socioeconómicos y culturales que son, en gran medida, los que afectan a los resultados. Por otro lado, en el proceso de atención primaria los resultados están influidos por las interacciones que otros niveles asistenciales tienen sobre el proceso de producción.

\subsection{Proceso de producción en atención especializada: el hospital}

Se realiza ahora un esfuerzo de conceptualización del proceso de producción de los servicios hospitalarios partiendo del concepto de hospital como organización multifuncional. Nuestra atención se centra en especificar los elementos que definen la producción eligiendo aquellos que ofrecen cierta garantía de que pueden ser imputados exclusivamente al hospital (independientemente de los factores ajenos que pueden influir en los resultados alcanzados).

El valor que se ha de generar a través de su proceso de producción es la salud, es decir la mejora de salud que tiene que experimentar el paciente. Desde este punto de vista, el proceso productivo empieza con la entrada del paciente enfermo y acaba cuando éste sale habiendo experimentado un cambio en su nivel de salud. Una vez admitido en el centro hospitalario, el paciente se transforma en el actor esencial en el proceso de producción del producto hospitalario.

Como se puede observar en la Figura 2, el paciente puede proceder del área sanitaria de referencia para el hospital o de cualquier otra área sanitaria (de dentro o fuera de España). Esta entrada (con o sin ingreso hospitalario) se produce por varios motivos: aquellos que son resultado de la intervención de una autoridad sanitaria (a, b, c y d) o resultado de la intervención directa del usuario (e y f). 


\section{Figura 2. El proceso productivo en el hospital general}

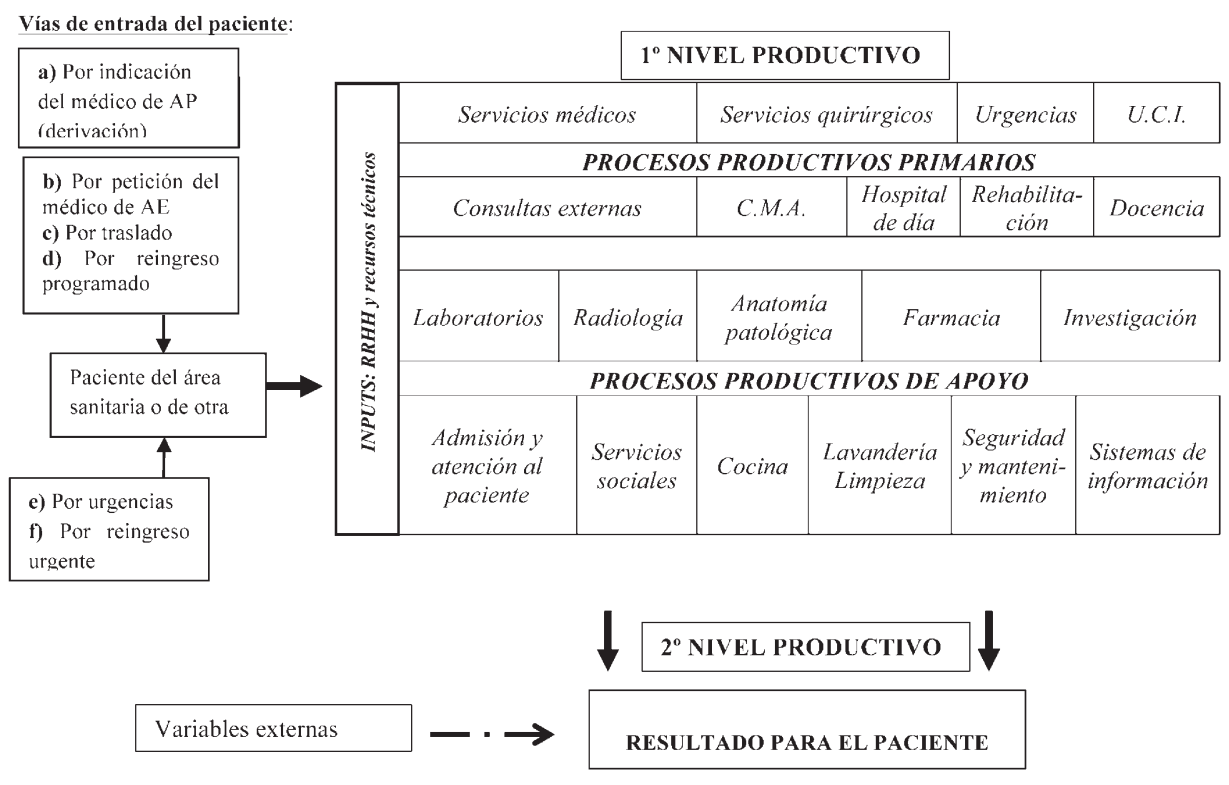

Fuente: elaboración propia a partir de Gorgemans (2013).

Así, entre los primeros motivos, situados en la parte superior de la gráfica se distinguen: a) por derivación desde la atención primaria; b) por petición del facultativo del centro de especialidades médicas en consultas externas; c) por traslado de otro hospital; d) por reingreso programado para seguir con el proceso terapéutico en el hospital. Esta primera vía da lugar al ingreso del paciente salvo que la intervención se realice en cirugía mayor ambulatoria. Cuando es el paciente que, por voluntad propia, se persona en el hospital lo hará siempre a través del servicio de urgencias del hospital distinguiéndose esta vez el carácter de la consulta realizada (o se persona por primera vez para una determinada patología o lo hace después de haber estado ingresado en el hospital por lo que se trataría de un reingreso urgente, respectivamente vías e y f). La entrada por el servicio de urgencias no da lugar forzosamente a una hospitalización. El proceso productivo resultara distinto para cada paciente y se diseña a medida que se avanza en el mismo.

Una vez iniciado el proceso se distinguen dos tipos de actividades, las de apoyo que pueden ser de tipo asistencial (radiología, análisis de laboratorio, farmacia) o no asistencial (cocina, lavandería, limpieza). Son en general actividades estandarizadas de poco contacto con el cliente, y servicios de masa. El resultado de los procesos de apoyo con carácter asistencial se utiliza como inputs en los procesos 
clínicos que se caracterizan por ser poco estandarizados y con alto contacto con el paciente. Estos servicios constituyen el núcleo del proceso productivo en el hospital y se identifican como procesos productivos primarios (Asociación Española de Contabilidad y Administración de Empresa, AECA, 1997). Estos procesos recogen todas las actividades realizadas directamente por los profesionales sanitarios en las distintas unidades de atención sanitaria y constituyen la función principal del hospital. Vienen definidos en función de la cartera de servicios del hospital y se distinguen, en el caso del hospital general, los servicios médicos, los servicios quirúrgicos, las urgencias y la Unidad de Cuidados Intensivos (UCI), las consultas externas, la Cirugía Mayor Ambulatoria (CMA), el Hospital de día y el servicio de rehabilitación. En cada uno de estos servicios se pueden realizar labores formativas del personal facultativo y sanitario no facultativo cuando el hospital tiene atribuciones de formación y docencia; no obstante, no se puede clasificar entre los procesos productivos de apoyo porque es parte integral del proceso asistencial y una forma de responder a la necesidad creciente de especialización y concentración de las actividades de los profesionales. Para cerrar la conceptualización del proceso productivo desde la óptica del hospital general como organización multi-servicio, se ha de considerar la producción de la investigación realizada en el centro hospitalario. De los resultados de los estudios empíricos llevados a cabo por los profesionales sanitarios se suelen beneficiar los pacientes para futuros procesos clínicos por lo que se puede considerar dicho proceso como un proceso de apoyo de naturaleza asistencial.

Los motivos que pueden dar lugar a la salida de un paciente y, por tanto, a la finalización del proceso productivo hospitalario, son también múltiples: a) el facultativo le puede dar el alta porque éste presenta una mejoría en su estado de salud; b) el paciente ha fallecido; c) el paciente ha sido trasladado a otro hospital de referencia; d) el paciente, después de consultar en el servicio de urgencias no está ingresado y vuelve a su domicilio; o, finalmente, e) el paciente pide el alta voluntaria.

La propuesta recogida en la Figura 2 recoge tres procesos (Bohígas, 1987): a) el proceso clínico que consiste en aplicar los conocimientos específicos al diagnóstico y tratamiento del paciente; b) el proceso técnico que consiste en producir una serie de productos que se utilizarán en el proceso clínico; y c) el proceso hotelero que se centra en la atención del paciente durante su estancia. Quizás se asemeje mucho más a la propuesta formulada por la AECA (1997) que divide el proceso del hospital en cuatro niveles: $1^{\circ}$ ) la estructura sanitaria que incluye los recursos humanos y técnicos; $2^{\circ}$ ) los procesos productivos de apoyo que recogen procesos asistenciales (radiología, los análisis en laboratorios, etc.); $3^{\circ}$ ) no asistenciales (limpieza, cocina, etc.); y $4^{\circ}$ ) los procesos productivos primarios (consultas, intervenciones quirúrgicas, etc.).

Igual que en atención primaria, en el primer nivel productivo se realizan los procesos productivos de apoyo y primarios para prestar la asistencia sanitaria al 
paciente y que éste pueda conseguir, en el segundo nivel productivo, el resultado que busca, que se concreta en el cambio del estado de salud del paciente o en el aumento de su calidad de vida.

Las cuestiones recogidas al explicar los procesos de producción de los dos niveles asistenciales ponen de manifiesto la dificultad para medir el producto obtenido. La diferenciación de dos niveles productivos en el proceso de producción nos permite centrar la atención en dos tipos de productos diferentes e interrelacionados: el output y el outcome. Conceptualmente es fácil establecer cuál es el producto final o resultado obtenido en el segundo nivel de producción. En este sentido, se puede decir que es la mejora que se produce en la salud del paciente. Desde el punto de vista de los pacientes esto es lo que se valora como producción ${ }^{6}$. El resultado final, outcome, es fácil de asumir como concepto, pero su medida es poco operativa para los estudios relativos a la gestión de los centros.

Desde el punto de vista de la gestión, es preciso conseguir medidas del producto que permitan evaluar el funcionamiento de los centros asistenciales. Para conseguirlo, únicamente se deben considerar aquellos factores que son controlables por los centros dejando fuera las variables externas ${ }^{7}$. Bajo esta perspectiva, la medida del producto se obtiene tomando como base los productos intermedios, poniendo el foco de atención en el primer proceso productivo propuesto en los dos niveles asistenciales, donde se consiguen los outputs, entendidos como las actividades realizadas para conseguir el producto final, pero considerando que la forma de realizar estas actividades tendrá una incidencia significativa en la salud del paciente, por tanto la calidad es un factor de suma importancia en la medida del producto.

\section{4. ¿CÓMO MEDIR EL PRODUCTO SANITARIO?}

En el estudio del output como medida válida para evaluar la forma de gestionar los servicios sanitarios debe tener en cuenta cuestiones como son la forma de cuantificarlo y, por supuesto, se debe incorporar la calidad de los servicios que se ofrecen.

En lo que se refiere a la cuantificación del producto intermedio en atención primaria, las unidades propuestas de manera más común han sido las consultas. Así, se cuantifican los servicios ofrecidos utilizando el número de consultas prestadas durante un determinado periodo de tiempo en un centro. Su utilización generalizada responde sobre todo a cuestiones relacionadas con la facilidad de

\footnotetext{
${ }^{6}$ Véase Carey y otros (1995).

${ }^{7}$ Véase Giuffrida y otros (1999).

${ }^{8}$ Véase la revisión de los principales trabajos sobre medida de la eficiencia en sanidad realizado por Puig y Dalmau (2000) y el trabajo de Romano y Choi (2016).
} 
cuantificación y disponibilidad de esta información. En los hospitales la forma de medir el producto intermedio es mediante los días de estancia, los ingresos y las intervenciones quirúrgicas. López-Casasnovas (2003) indica que estas actividades pueden ser válidas a nivel macroeconómico para medir la eficiencia de los hospitales pero actividades como la investigación o la docencia, que tienen externalidades positivas, no pueden ser identificadas con un beneficio inmediato.

Esta forma de medir el producto no está exenta de limitaciones, una de las más importantes es la dispersión en la complejidad de los problemas de salud de los pacientes tratados en los diferentes servicios sanitarios. Para realizar procesos de evaluación efectivos es necesario tratar de paliar este problema, para ello se pueden utilizar sistemas que permiten la clasificación de los pacientes atendidos en los servicios sanitarios, se trata de los sistemas de ajuste de riesgo que agrupan pacientes por consumo de recursos similares y que son herramientas muy útiles en el análisis del funcionamiento de los centros sanitarios. Estos sistemas de ajuste por riesgo permiten ajustar la medida del producto para, de esta forma, conseguir una mayor aproximación a la realidad del centro asistencial.

En atención primaria es muy compleja la propuesta de un sistema de ajuste, debido a que en este entorno existe una enorme diversidad de servicios mucho más variada que en la asistencia en hospitales (Gold, 1988). En la mayoría de los casos los pacientes presentan un diagnóstico claro, sobre el cual se suman problemas sanitarios, problemas sociales y psicológicos. Por tanto el abanico de asistencia que se debe ofrecer es muy amplio, y para poder aplicar, en este ámbito, los métodos de ajuste de riesgo, se debe realizar un gran esfuerzo de abstracción que nos permita realizar una agrupación de pacientes (Johnson, 1989; Hutchinson y otros, 1991; y Hutchinson y Fowler, 1992).

Entre los sistemas de ajuste por riesgo en la atención primaria, es el Grupo Clínico Ajustado (GCA), propuesto por Starfield y otros (1991) y Weiner y otros (1991) con el que se ha trabajado para su aplicación en nuestro país (Juncosa y Bolívar, 1997). Sin embargo, existen dificultades para utilizar estos métodos en un entorno diferente para el que fueron diseñados (Sicras-Mainar y otros, 2007; Sicras-Mainar y otros, 2010). En cualquier caso, para su adaptación y aplicación a nuestro entorno es necesario contar con un conjunto básico normalizado de datos tal como plantea Fuste y otros (2002). Hasta el momento se han realizado algunas aplicaciones prácticas, como la realizada por Simar-Mainar y otros (2010) en las que se han encontrado ciertas dificultades, como la complejidad y variabilidad de la actividad realizada en primaria para definir una unidad de medida, pero a pesar de estas limitaciones los autores consideran que este sistema de clasificación puede servir para ayudar a la gestión y a tomar las decisiones de financiación en atención primaria. Esta misma línea se sigue en la propuesta del plan de renovación de la atención primaria del Servicio Andaluz de Salud (2016), en la que se manifiesta que los GCA han demostrado su capacidad explicativa en el uso de 
los recursos y los resultados clínicos en la atención primaria andaluza. Por tanto, son un sistema de clasificación interesante y que puede servir para conseguir un mayor ajuste a la realidad de la actividad realizada en los centros de atención primaria, lo cual permite mejorar sustancialmente los resultados derivados de los análisis sobre el funcionamiento de los centros de atención primaria.

En el segundo nivel asistencial, en los hospitales del Sistema Nacional de Salud desde el año $1991^{9}$ se ha generalizado la implantación tanto de la codificación del Conjunto Mínimo Básico de Datos (C. M. B. D) y el desarrollo de los Grupos de Diagnóstico Relacionado (GRD). Los consumos de recursos son medidas relativas que se identifican actualmente en función del proceso asistencial (GRD clasificación de los pacientes en grupos homogéneos con relación a la utilización de recursos y significativos, es decir, con lógica clínica, para los médicos) y permiten establecer comparaciones entre los hospitales sobre todo en cuanto se trata de los costes soportados para un determinado proceso.

Además de la cuantificación de la actividad y del ajuste por riesgo es importante tener en cuenta la calidad con la que se presta el servicio asistencial. Esta variable está presente en la agenda de muchos sistemas de salud en el mundo y gran parte de este interés se ocasiona en base a las transformaciones de los sistemas de salud, acompañados de la creación de nuevas estructuras organizativas y de una orientación mayor hacia el paciente.

La OMS (2006) sugiere que cualquier sistema de salud debe buscar mejorar la calidad respeto a seis dimensiones. Estas dimensiones requieren que el servicio sanitario sea: eficaz, eficiente, accesible, aceptable (orientado al paciente), equitativo y seguro ${ }^{10}$. Los atributos habitualmente citados en la bibliografía, por autores o instituciones como Palmer (1983), Donabedian (1988), Maxwell (1992), Consejo de Europa (1998), NHS (1997), Joint Commission for the Accreditation of Health Care Organizations (JCAHO, 1999) o el Instituto de Medicina (IOM, 2001), incluyen (por orden decreciente de frecuencia), entre otros: efectividad, eficiencia, acceso, competencia técnica, equidad, adecuación, disponibilidad, seguridad, y continuidad ${ }^{11}$.

La Unión Europea (Aibar, 2005) recomienda a sus estados miembros el desarrollo y puesta en marcha de sistemas de mejora de la calidad en los servicios de salud. En España, tanto la LGS (1986) como la Ley 16/2003, de 28 de mayo, de

\footnotetext{
${ }^{9}$ Análisis y desarrollo de los GDR en el Sistema Nacional de Salud (1999). Ministerio de Sanidad y Consumo.

${ }^{10}$ Los beneficios económicos de la seguridad del paciente se miden en términos de disminución de la estancia media, de los costes derivados de los juicios, etc. que llegan a un importe de 6 a 29 billones de \$ según países (WHO, 2006).

${ }^{11}$ Una dimensión primordial únicamente mencionada por Maxwell (1992) su relevancia estriba en que se refiere al patrón general y balance de servicios óptimos que podrían alcanzarse, teniendo en cuenta las necesidades y carencias de la población como un todo.
} 
Cohesión y Calidad del Sistema Nacional de Salud (LCC), recogen una de las características fundamentales del sistema de salud español: "la prestación de una atención integral de la salud procurando altos niveles de calidad debidamente evaluados y controlados". El Ministerio de Sanidad define un conjunto de seis grandes áreas de trabajo del Plan de Calidad que pretenden dar respuesta a los principales retos del sistema de salud: protección, promoción de la salud y prevención; fomento de la equidad; apoyo a la planificación y el desarrollo de los recursos humanos en salud; fomento de la excelencia clínica; utilización de las tecnologías de la información para mejorar la atención a los ciudadanos; y, aumento de la transparencia (Plan de Calidad para el SNS 2014). En la Tabla 2 se muestra el resumen de los principales atributos de la calidad asistencial.

\section{Tabla 2. Atributos de la calidad asistencial en el SNS}

\begin{tabular}{|l|l|}
\hline \multicolumn{1}{|c|}{ Atributo } & \multicolumn{1}{c|}{ Definición } \\
\hline Efectividad & $\begin{array}{l}\text { Capacidad de un procedimiento concreto de mejorar el grado de salud. } \\
\text { Es también la relación entre el impacto real de un servicio y su impacto } \\
\text { potencial en situación ideal. Relaciona, igualmente, la capacidad de } \\
\text { actuar del médico y su equipo, de manera que los pacientes consigan } \\
\text { el resultado más deseable. Incluye también minimizar los riesgos y un } \\
\text { sistema de documentación del estado de salud y de la atención prestada. }\end{array}$ \\
\hline Eficiencia & $\begin{array}{l}\text { Relación entre el impacto real de un servicio y su coste de producción. } \\
\text { También se conceptúa como la prestación del máximo de servicios de } \\
\text { calidad por unidad de recursos disponibles o la prestación del máximo } \\
\text { de unidades comparables de cuidados sanitarios por unidad de recursos } \\
\text { utilizados. }\end{array}$ \\
\hline Accesibilidad & $\begin{array}{l}\text { Facilidad con que se obtienen, en cualquier momento, los cuidados } \\
\text { médicos, a pesar de posibles barreras económicas, geográficas, de } \\
\text { organización, psíquicas y culturales. }\end{array}$ \\
\hline Competencia & $\begin{array}{l}\text { Capacidad del profesional de utilizar plenamente sus conocimientos } \\
\text { en su tarea de proporcionar salud y satisfacción a los usuarios. Se } \\
\text { profesional } \\
\text { refiere tanto a la función operacional como a la relacional y tanto al } \\
\text { profesional sanitario como a la organización. }\end{array}$ \\
\hline Equidad & $\begin{array}{l}\text { Requiere que todos los usuarios sean tratados igual cuando tienen } \\
\text { necesidades comunes, y que sus diferencias se aborden (por ejemplo por } \\
\text { cuestiones de género) de manera diferenciada. Salvaguardar un trato } \\
\text { igualitario entre las regiones. }\end{array}$ \\
\hline Disponibilidad & $\begin{array}{l}\text { Relación entre la disponibilidad de los servicios y las necesidades de la } \\
\text { población. Su medida comprende tanto la cantidad de recursos como su } \\
\text { distribución geográfica. }\end{array}$ \\
\hline $\begin{array}{l}\text { Grado en que los servicios sanitarios se hallan en estado operativo. Los } \\
\text { cuidados médicos deben ser disponibles cuando se necesiten durante el } \\
\text { ciclo de vida de la persona y cuando surja cualquier enfermedad. }\end{array}$ \\
\hline Edecuacion
\end{tabular}




\begin{tabular}{|l|l|}
\hline \multicolumn{1}{|c|}{ Atributo } & \multicolumn{1}{c|}{ Definición } \\
\hline Seguridad & $\begin{array}{l}\text { Balance positivo de la relación beneficios/riesgos. También puede } \\
\text { conceptuarse como el resultado de la acción sinérgica de la } \\
\text { competencia y trato del personal sanitario. Respecto a los equipos y } \\
\text { edificios, la seguridad se refiere a la garantía y protección de los mismos. }\end{array}$ \\
\hline Aceptabilidad & $\begin{array}{l}\text { Incluye dos dimensiones: satisfacción del paciente (cumplimiento de } \\
\text { sus expectativas en cuanto a la atención recibida y los resultados) y } \\
\text { Satisfacción con la organización, la asistencia recibida, los profesionales } \\
\text { y los resultados de la asistencia sanitaria. }\end{array}$ \\
\hline Continuidad & $\begin{array}{l}\text { Tratamiento del paciente como un todo en un sistema de atención } \\
\text { integrado. Los servicios deben ser continuos y coordinados para que el } \\
\text { plan de cuidados de un enfermo progrese sin interrupciones. }\end{array}$ \\
\hline $\begin{array}{l}\text { Satisfacción del } \\
\text { profesional }\end{array}$ & $\begin{array}{l}\text { Grado de satisfacción del profesional sanitario con las condiciones y } \\
\text { desarrollo del trabajo. Su inclusión entre los atributos de la calidad se deriva } \\
\text { de la influencia que tiene la satisfacción laboral en la calidad del trabajo. }\end{array}$ \\
\hline
\end{tabular}

Fuente: Gorgemans (2013).

Donabedian (1984) distingue tres aspectos diferenciados en la calidad de los servicios sanitarios, el aspecto técnico que se mide en términos únicamente médicos o calidad técnica; el aspecto individualizado que consiste en que sea el propio individuo quién valore sus estados de salud en función de su disposición a aportar dinero y tiempo para mejorarlos; y, finalmente, el aspecto social de la calidad que aparece cuando la asistencia es pagada por la sociedad e incorpora, por tanto, la valoración social de los costes de la asistencia. Los dos últimos aspectos en esta propuesta tienen en cuenta el valor otorgado a la salud, aunque los criterios que se incorporan para la valoración sean distintos. En este trabajo el interés se centra en el análisis de la producción en sanidad y la calidad derivada del proceso de producción del sistema sanitario, por lo que interesa poner la atención en los aspectos de calidad en la prestación de servicios que recibe el paciente (incorporados en el proceso productivo) como resultado de la actuación directa de los profesionales sanitarios (recursos en el proceso productivo).

La OCDE reconoce a la calidad técnica la ventaja de proporcionar el valor que genera un sistema de asistencia sanitaria (OCDE, 2010). Sobre esta base se han desarrollado numerosos índices de calidad que han sido probados y confirmados como fiables, válidos, de uso fácil y útiles para aumentar el nivel de calidad. A posteriori permiten comparaciones entre proveedores y entre países mientras se haya introducido un sistema de ajuste por riesgo.

Los indicadores de calidad técnica pueden clasificarse en dos grupos principales: conjunto de inputs o acciones que conducen a mejores resultados, es decir, medidas de estructura y proceso en el sentido clásico de medida de la calidad de Donabedian (1966) y medidas directas de resultados. En la Tabla 3, se proponen posibles dimensiones de medición de la calidad técnica con algunos ejemplos de indicadores y sus dimensiones cuantitativas. 


\section{Tabla 3. Dimensiones de la calidad técnica}

\begin{tabular}{|c|c|c|c|}
\hline $\begin{array}{c}\text { Aspectos a } \\
\text { valorar }\end{array}$ & $\begin{array}{l}\text { Dimensión de la } \\
\text { calidad }\end{array}$ & Ejemplos de Indicadores & $\begin{array}{c}\text { Ejemplos de Dimensiones } \\
\text { cuantitativas }\end{array}$ \\
\hline Estructura & $\begin{array}{l}\text { Cómo se distribu- } \\
\text { yen los recursos en } \\
\text { términos de tiem- } \\
\text { po, lugar y recepti- } \\
\text { vidad a las necesi- } \\
\text { dades de las } \\
\text { poblaciones (acce- } \\
\text { so). Ecuanimidad } \\
\text { en compartir cos- } \\
\text { tes y beneficios } \\
\text { (equidad). }\end{array}$ & $\begin{array}{l}\text { Acceso geográfico, acceso } \\
\text { arquitectónico, Plan de ca- } \\
\text { tástrofes, tratamiento de } \\
\text { residuos. } \\
\text { Listas de espera, tiempo de } \\
\text { espera en urgencias, etc. } \\
\text { Costes, Nivel de forma- } \\
\text { ción del personal, Habili- } \\
\text { dad técnica. }\end{array}$ & $\begin{array}{l}\text { Listas de espera quirúrgicas } \\
\text { del SNS oscilan entre un } \\
\text { tiempo medio máximo de } \\
160 \text { días para Neurocirugía } \\
\text { y mínimo de } 52 \text { días en Der- } \\
\text { matología; la lista de espe- } \\
\text { ra en consultas externas de } \\
58 \text { días de media entre los } \\
\text { servicios (Informe Minis- } \\
\text { terio de Sanidad y Servi- } \\
\text { cios sociales, diciembre } \\
2015 \text { ). }\end{array}$ \\
\hline Procesos & $\begin{array}{l}\text { Cómo se aplican } \\
\text { los recursos (efi- } \\
\text { ciencia). } \\
\text { Uso de tiempo y } \\
\text { recursos (eficien- } \\
\text { cia). } \\
\text { Evitar el despilfa- } \\
\text { rro (eficiencia). } \\
\text { Reducción del } \\
\text { riesgo (seguridad). } \\
\text { Práctica basada en } \\
\text { la evidencia (ade- } \\
\text { cuación). } \\
\text { Atención centra- } \\
\text { da en el paciente } \\
\text { (continuidad). } \\
\text { Información al pa- } \\
\text { ciente/público } \\
\text { (elección, transpa- } \\
\text { rencia, responsabi- } \\
\text { lidad). }\end{array}$ & $\begin{array}{l}\text { Uso de los medicamentos } \\
\text { Índice ocupación quirófa- } \\
\text { nos; } \mathrm{N}^{\circ} \text { de pacientes pen- } \\
\text { dientes de } 1^{\mathrm{a}} \text { consulta; de- } \\
\text { mora prospectiva en } \\
\text { consultas externas; \% de } \\
\text { pacientes que permanecen } \\
\text { más de } 6 \text { h. en urgencia } \\
\text { Gestión de riesgos, infec- } \\
\text { ciones, caídas, complica- } \\
\text { ciones. } \\
\text { Continuidad entre la aten- } \\
\text { ción primaria y el hospital } \\
\text { Relación interpersonal } \\
\text { con el paciente, continui- } \\
\text { dad de la asistencia } \\
\text { Comunicación con los } \\
\text { clientes y el resto de los } \\
\text { stakeholders }\end{array}$ & $\begin{array}{l}\text { Los índices de ocupación } \\
\text { tienen mucha utilidad } \\
\text { prospectiva para planificar } \\
\text { y evaluar la programación } \\
\text { quirúrgica de un centro } \\
\text { hospitalario y daría una } \\
\text { medida directa del rendi- } \\
\text { miento* Una medida } \\
\text { aceptable sería el } 80 \% \text { de } \\
\text { tasa de ocupación neta. } \\
\text { Los datos publicados en el } \\
\text { CMBD permiten calcular } \\
\text { niveles de calidad por hos- } \\
\text { pital en eventos adversos. } \\
\text { Por ejemplo, en 2013, la } \\
\text { incidencia intrahospitala- } \\
\text { ria ajustada al riesgo por } \\
\text { cada 1.000 pacientes a } \\
\text { riesgo en mayores de } 18 \\
\text { años por infección nosoco- } \\
\text { mial: } 14,9 \text {; sepsis: } 5,3 \text {; epi- } \\
\text { so-dio de tromboembolis- } \\
\text { mo tras IQ: } 7,8 \text {. }\end{array}$ \\
\hline
\end{tabular}

*Serra Sutton, V. y otros (2011): "Eficiencia en la utilización de los bloques quirúrgicos. Definición de indicadores", Madrid: Plan de Calidad para el Sistema Nacional de Salud. Ministerio de Ciencia e Innovación. Barcelona: Agència d'Informació, Avaluació i Qualitat en Salut. 


\begin{tabular}{|c|c|c|c|}
\hline $\begin{array}{l}\text { Aspectos a } \\
\text { valorar }\end{array}$ & $\begin{array}{l}\text { Dimensión de la } \\
\text { calidad }\end{array}$ & Ejemplos de Indicadores & $\begin{array}{l}\text { Ejemplos de Dimensiones } \\
\text { cuantitativas }\end{array}$ \\
\hline Resultados & $\begin{array}{l}\text { Salud de la pobla- } \\
\text { ción (mejora de la } \\
\text { salud). } \\
\text { Resultado clínico } \\
\text { (efectividad). } \\
\text { Satisfacer expecta- } \\
\text { tivas del público y } \\
\text { del personal (coste- } \\
\text { beneficio). }\end{array}$ & $\begin{array}{l}\text { Mortalidad, morbilidad, dis- } \\
\text { capacidad y calidad de vida } \\
\text { Confección de guías, Grado } \\
\text { de variabilidad en la práctica } \\
\text { médica. } \\
\text { Satisfacción del paciente, } \\
\text { satisfacción del trabajador, } \\
\text { satisfacción del resto de los } \\
\text { stakeholders. }\end{array}$ & $\begin{array}{l}\text { AVAC (años de vida ajusta- } \\
\text { dos por calidad) y AVAD } \\
\text { (años de vida ajustados por } \\
\text { discapacidad) son términos } \\
\text { corrientes para valorar el } \\
\text { riesgo y beneficio. Una in- } \\
\text { tervención que proporciona } \\
\text { durante dos años adicionales } \\
\text { un estado de salud de 0,5 } \\
\text { equivaldría a un AVAC. } \\
\text { Tasa media de mortalidad } \\
\text { por infarto de miocardio por } \\
\text { edad } \geq 40 \text { años (2013): } 70,21 \\
\text { Los resultados de estudio de } \\
\text { calidad del servicio y satis- } \\
\text { facción son públicos en Ca- } \\
\text { taluña y en Andalucía (véa- } \\
\text { se memorias de los servicios } \\
\text { regionales y página web del } \\
\text { SAS: http://www.juntadean- } \\
\text { dalucia.es/servicioandaluz- } \\
\text { desalud/indicadores/). }\end{array}$ \\
\hline
\end{tabular}

Fuente: elaboración propia a partir de Gorgemans (2013).

Las primeras medidas de calidad técnica, basadas en la estructura, recogen las características del proveedor sanitario y la idoneidad de los factores empleados en la producción de los servicios (camas hospitalarias, facultativos y profesionales no facultativos). Las medidas de calidad sobre los procesos reflejan si se siguen prácticas universalmente aceptadas y basadas en datos contrastados (en términos de prevención sobre todo a través de la vacunación, o control de la tensión arterial, etc.). Las medidas de calidad técnica que se apoyan en los resultados capturan la mejora (o deterioro) de la salud en relación con la atención médica.

Las medidas de calidad técnica combinadas con los datos sobre uso de recursos pueden emplearse para obtener indicadores de la eficiencia de un sistema de asistencia sanitaria o de la rentabilidad que genera. Para ello, se debe considerar un sistema de ajuste de riesgo para atender a las características de la población de la zona de referencia de cada uno de los proveedores sanitarios. Esta línea de trabajo viene siendo impulsada por la OCDE desde el año 2004, (informe "Health at a Glance" con un capítulo dedicado a la calidad sanitaria), 
resultado del proyecto Health Care Quality Indicators (HCQI ${ }^{12}$ cuyo objetivo era elaborar un conjunto de indicadores de calidad de los servicios sanitarios (Mattke y otros, 2006; García-Armesto y otros, 2007) que permitiera comparaciones internacionales y completara así los esfuerzos realizados por los organismos nacionales e internacionales ${ }^{13}$. Este tipo de valoración de la calidad puramente clínica ha sido igualmente desarrollado ${ }^{14}$ en otros países. Así, en Estados Unidos, se creó, en 1989, una agencia de seguimiento de la calidad bajo el nombre Agency for Healthcare Research and Quality (AHRQ) cuya misión es medir la calidad, la seguridad, y la eficacia del sistema sanitario para la población americana. La agencia americana utiliza indicadores como los Prevention Quality Indicators (PQI), los Inpatient Quality Indicators (IQI) y los Patient Safety Indicators (PSI).

Como recogen De los Santos Ichaso y Garcia-Armesto (2012), las organizaciones internacionales han utilizado dos tipos de indicadores para evaluar y comparar la calidad de los cuidados de atención primaria de forma indirecta, por un lado, aproximando la efectividad de la promoción de la salud y la prevención mediante tasas poblacionales de obesidad, tabaquismo, enfermedades prevenibles mediante vacunación (hepatitis, sarampión, rubéola, parotiditis, difteria) y abortos y, por otro lado, se trata de medir la efectividad diagnóstica. Este segundo indicador de calidad técnica en el proceso de producción de atención primaria se refiere principalmente a su capacidad de resolución, uno de los objetivos claves de este primer nivel asistencial. En este sentido el indicador PQI también conocido como Ambulatory Care Sensitive Conditions (ACSC), recoge hospitalizaciones que identifican problemas de salud susceptibles de mejores cuidados en atención primaria y que podrían ser evitados si esos cuidados mejoran. Es decir se centran en valorar cómo se resuelven los problemas de salud en los centros de atención primaria. Algunas propuestas para medir el nivel resolutivo de los centros de atención primaria han considerado como una aproximación las derivaciones realizadas hacia la atención especializada ${ }^{15}$. Las derivaciones pueden ser utilizadas como factor de calidad por las propias características de la atención primaria, dado que funciona como filtro de paso hacia la atención especializada. Por tanto, las derivaciones proporcionan información sobre los problemas de salud que no

\footnotetext{
${ }^{12}$ Para más información consultar la página web: y Foundation for Accountability. http://www. markle.org/resources/facct/index.php.

${ }^{13}$ Habiéndose confirmado su validez científica, verificado su importancia en términos clínicos y políticos y su viabilidad.

${ }^{14}$ Véase Agency for Healthcare Research and Quality (AHRQ) para un censo completo de las organizaciones que han desarrollado indicadores de calidad. http://www.ahrq.gov

${ }^{15}$ Véase Ortún y otros (1999).
} 
se resuelven en primaria y pasan a especializada ${ }^{16}$. Al mismo tiempo constituyen el nexo de unión entre los dos niveles de asistencia sanitaria. La existencia de derivaciones innecesarias desde primaria supone el incumplimiento de uno de sus principales objetivos, lo cual conlleva que desde la perspectiva económica se produzca una utilización innecesaria de recursos sanitarios y que desde el punto de vista del paciente se sufran costes personales que se traducen en insatisfacción. Existen algunos estudios sobre las derivaciones desde atención primaria como el realizado por García Olmos y otros (1994), Peiró (2008) y Espinoza y otros (2015), en los que se detecta una gran variabilidad de las derivaciones entre profesionales, lo que lleva a los autores a plantearse la existencia de derivaciones innecesarias en atención primaria ${ }^{17}$. Por tanto una medida más ajustada sería, la comentada anteriormente, el ACSC, estos indicadores centran en el estudio de la ratio de admisión hospitalaria ${ }^{18}$. Los ingresos por ACSC podrían haber sido resueltos con una buena asistencia primaria (Caminal, 2003). Así que podría esperarse que una atención primaria efectiva redujera el número de admisiones en hospitales por enfermedades crónicas, como puede ser en el caso del asma o de la diabetes. Una buena prevención y un seguimiento adecuado de estas enfermedades desde la atención primaria podrían evitar la hospitalización ${ }^{19}$. De manera que este indicador de hospitalización se presenta como un "aviso" del estado de la atención primaria (Gervás y Caminal, 2007). Sería una variable importante para considerar la evaluación de la calidad técnica con la que funciona la atención primaria en un determinado momento.

Los indicadores PSI y IQI son una opción interesante para conocer la calidad técnica derivada del proceso de producción en el hospital. Los PSI permiten cuantificar las complicaciones potenciales "intra-hospitalarias" y eventos adversos después de cirugías o de procedimientos, la validación ha demostrado que los indicadores de seguridad del paciente tienen una notable capacidad para clasificar de forma apropiada eventos adversos ${ }^{20}$.

Se ha valorado también muy positivamente la utilización de los IQI como tasas de mortalidad en determinados tipos de patología, como indicadores de

\footnotetext{
${ }^{16}$ La admision en el hospital puede proporcionar infromación sobre el funcionamiento de atención primaria (Jankowski, 1999).

${ }^{17}$ Véase también el trabajo de Ortún y otros (1999).

${ }^{18}$ Véase Billings y otros, (1996), Begley y otros (1994), Aveyard (1997) y Casanova y otros (1996).

${ }^{19}$ En el trabajo de Romano y Choi (2016) se utiliza esta variable de calidad para medir la eficiencia en atención primaria.

${ }^{20}$ Estos eventos adversos son bastante infrecuentes. No obstante, la definición validada en España (MSPS, 2008) resultó ser diferente de la definición utilizada por la OCDE (2009) para los indicadores de UPP (úlcera por presión), tromboembolismo pulmonar-trombosis venosa profunda, parto con instrumentación y parto sin instrumentación.
} 
base hospitalaria ajustados por riesgo. Este indicador es una medida de resultado tal y como lo plantean Shaw y Kalo (2002) y Capella (2000). La mortalidad en los procedimientos considerados puede ser aceptada como signo de mala calidad. Pero considerando siempre la mortalidad por causas determinadas (los IQI) y al ajuste de dichas tasas por diferentes variables como pueden ser la comorbilidad y especialmente el uso del índice de Charlton (Charlton y otros, 1983), o una corrección por la edad de los pacientes atendidos, o por diferentes índices de severidad o de casuística, etc. (Gil, 2005) ${ }^{21}$. La mortalidad evitable experimenta gran variabilidad territorial y presenta una relación negativa con diversos factores socioeconómicos (López y Rico, 2001; Ortún y Gisper, 1988; e Informe SESPAS 1995, 2000). En consecuencia se suele usar causas de muerte que permiten valorar la calidad de las intervenciones sanitarias y accesibilidad a los servicios sanitarios (Westerling, 2003 y Simonato y otros, 1998) en lugar de valorar la efectividad de las políticas de salud y la asignación de recursos (Holland y otros, 1994), evitando el peso de otros factores como pueden ser los medioambientales o socioeconómicos sobre la salud de la población. Gispert y otros (2006) han establecido una lista de consenso adaptada al contexto sanitario español.

Otros indicadores para medir la calidad de los resultados son los reingresos, habitualmente propuestos para valorar la calidad de los cuidados. El reingreso es cualquier ingreso hospitalario precedido en el tiempo por un alta y que siempre ocurre con anterioridad a los 30 días desde el alta del paciente y siempre por la misma causa, es decir, el mismo diagnóstico de entrada que en el caso del episodio anterior (misma Categoría Diagnóstica Mayor). No obstante, una relación consistente entre reingresos y calidad no ha sido establecida (Weissman y otros, 1999). Esta variable podría ser considerada como señal de una merma de la calidad asistencial cuando se trata de unas determinadas patologías (Frankl y otros, 1991 y Thomas, 1996) o en aquellas enfermedades de alta cronicidad (Weissman y otros, 1999; Ashton y otros, 1995) pero, en el resto de los casos, se ha puesto en duda su utilización como índice de calidad (Benbassat y otros, 2000; Clark, 1990). Según el INSALUD ${ }^{22}$, la monitorización de los reingresos forma parte de los indicadores centinelas propuestos para evaluar los resultados de la asistencia sanitaria, junto a la mortalidad y las complicaciones.

Se resumen, en la siguiente tabla, y para ambos niveles de atención sanitaria, las distintas variables que se podrían tener en cuenta a la hora de valorar el producto sanitario.

\footnotetext{
${ }^{21}$ En los PSI se usan también.

${ }^{22}$ Plan de Calidad, SNS, 2001.
} 
Tabla 4. Propuesta de variables útiles para la medición del producto sanitario

\begin{tabular}{|l|l|l|}
\hline \multicolumn{1}{|c|}{ Medida } & \multicolumn{1}{|c|}{ Atención primaria } & \multicolumn{1}{c|}{ Atención especializada } \\
\hline $\begin{array}{l}\text { OUTPUT AJUSTADO POR } \\
\text { RIESGO }\end{array}$ & Consultas x GCA & $\begin{array}{l}\text { Altas x peso medio } \\
\text { GRD, ingresos, estancias, } \\
\text { intervenciones quirúrgicas, } \\
\text { urgencias, consultas externas, } \\
\text { pruebas laboratorio, pruebas } \\
\text { por imagen. }\end{array}$ \\
\hline $\begin{array}{l}\text { Aproximación al OUTCOME } \\
\text { (ponderando por la calidad) }\end{array}$ & $\begin{array}{l}\text { Efectividad de la promoción y yención de la salud } \\
\text { prevendidad } \\
\text { Efectividad diagnóstica: } \\
\text { ACSC }\end{array}$ & $\begin{array}{l}\text { Indicadores de seguridad: } \\
\text { frecuencia de eventos } \\
\text { adversos y tasa de mortalidad } \\
\text { en determinadas patologías } \\
\text { ajustadas por edad, sexo y } \\
\text { comorbilidades de Elixhauser. } \\
\text { Reingresos en la misma } \\
\text { categoría diagnóstica mayor. }\end{array}$ \\
\hline
\end{tabular}

Fuente: elaboración propia.

\section{CONCLUSIONES}

Dentro del Sistema Nacional de Salud se han introducido cambios organizativos y diferentes fórmulas de gestión, con el propósito de conseguir mayores niveles de descentralización y potenciar una separación entre las funciones de financiación, compra y provisión. Todas las reformas que se han puesto en marcha a lo largo de estos últimos 30 años en nuestro sistema sanitario se han justificado principalmente por la contención del incremento del gasto sanitario y la sostenibilidad del estado de bienestar. Este proceso de cambio en el que está inmerso el sistema sanitario conlleva una mayor descentralización lo que requiere más y mejor información; información que debe ser homogénea, objetiva y útil para utilizarla en los procesos de evaluación y control que permitan tomar decisiones encaminadas hacia la mejora continua del sistema sanitario.

La utilización de herramientas que permitan obtener una evaluación del funcionamiento de los servicios sanitarios y de la actividad asistencial que ofrecen no está exenta de limitaciones, derivadas en buena medida de la delimitación y medida del producto sanitario. En este trabajo se aborda una reflexión sobre la conceptualización del producto sanitario, desde la aproximación al sector servicios, lo que nos permite conocer algunas de las principales características como son la intangibilidad y la intervención del paciente en el proceso de producción, lo que podría considerarse incluso una co-producción del propio paciente, estas 
características dificultan la definición del producto y su medida. Para tratar de paliar estas dificultades y acercarnos al concepto de producto sanitario es conveniente conocer cómo se desarrolla el proceso de producción en el ámbito sanitario, diferenciando entre los dos niveles asistenciales. Esto se debe a que los pacientes que se atienden presentan características diferentes y, por tanto, los procesos de producción son diferentes, lo que justifica que se haya optado por presentar de forma separada los procesos de producción de los dos niveles asistenciales: la atención primaria y la atención especializada en los hospitales. Pero pese a estas diferencias se observa que lo que marca el inicio de los procesos de producción, tanto en primaria como en el hospital, es la entrada o demanda del paciente de servicios sanitarios. En ambos procesos de producción el valor que se pretende conseguir es mejorar la calidad de vida del paciente o de su salud, para conseguirlo se puede diferenciar dentro de proceso dos niveles productivos. En el primer nivel productivo se combinan una serie de recursos humanos y técnicos para ofrecer a los pacientes los servicios asistenciales que precisen, en este primer nivel productivo se produce el producto intermedio (output). En el segundo nivel productivo los productos intermedios se convierten en los recursos necesarios para conseguir el resultado (outcome) sobre la salud del paciente. Este análisis del proceso de producción nos permite centrar nuestro interés en el primer nivel productivo debido a que el resultado que se consigue en el segundo nivel de producción está influido por variables externas, como son el estilo de vida del paciente y factores socioeconómicos y culturales. De forma que para el analizar la gestión sobre el funcionamiento de los centros asistenciales es pertinente centrarnos en el producto intermedio y determinar la forma de obtener una medida válida que permita evaluar y proponer mejoras en la forma de gestionar los servicios sanitarios.

La medida del producto intermedio sanitario sigue siendo complicada, en los dos niveles asistenciales, como era de esperar, los recursos y los outputs realizados son diferentes. Pero para abordar la forma de medir el output en ambos casos se mantienen en común cuestiones sustanciales. Se propone medir el producto utilizando las medidas de la actividad de asistencia sanitaria prestada en los diferentes centros sanitarios, pero debido a las particularidades de este tipo de servicios, donde el paciente y las características que éste presenta juegan un papel relevante en el proceso de producción, es necesario que la medida de la actividad realizada se ajuste utilizando la grupos de pacientes atendidos mediante los sistemas de ajuste por riesgos. En los hospitales se utilizan desde el año 1991 los grupos de diagnóstico relacionado (GRD), sin embargo en atención primaria, hasta estos últimos años, no existía un consenso sobre el método de ajuste que mejor se aproximaba a nuestra atención primaria, pero hemos visto como en los trabajos más recientes se propone el Grupo Clínico Ajustado (GCA) como un sistema de clasificación útil para ayudar a la gestión y financiación de nuestra atención primaria. 
La propuesta de medida del producto no solo pasa por contar con una medida de actividad ajustada a las características de los pacientes que se atienden también se debe recoger medidas de la calidad sobre la forma de prestar esta actividad, principalmente por la influencia directa que la calidad de los servicios puede tener sobre el resultado final que consiga el paciente. La calidad es una variable que se encuentra presente en las agendas de los sistemas sanitarios, en este trabajo se recogen una propuesta sobre los atributos asistenciales que debe reunir los sistemas sanitarios. Sin embargo en el argumento que sigue este trabajo nos interesa proponer variables que permitan recoger la calidad con la que se ofrece la asistencia sanitaria, es decir, la propuesta de indicadores que puedan medir la calidad técnica sobre el desarrollo del proceso asistencial. En la atención primaria, como se ha visto, una propuesta interesante como indicador para evaluar la calidad técnica del funcionamiento de los centros de salud sería los Ambulatory Care Sensitive Conditions (ACSG). En los hospitales, algunos de los indicadores de calidad técnica son los PSI, que permiten conocer las complicaciones o eventos adversos después de cirugías, en este sentido las infecciones nosocomiales, se aceptan como representativas del nivel de calidad técnica de los proceso en los hospitales. Otro de los indicadores son los IQI, como tasas de mortalidad evitable. Otro de los indicadores propuestos son los reingresos, como una señal de merma de la calidad.

En este trabajo se ha realizado una reflexión sobre todas las cuestiones relevantes a la hora de hablar del proceso de producción en el ámbito sanitario y se propone la forma de medir el producto, mediante la integración de medidas de actividad ajustadas por riesgo con medidas de la calidad técnica del servicio prestado, que se tendrían que considerarse en sanidad para poder realizar análisis sobre el funcionamiento de los centros que prestan asistencia sanitaria.

\section{BIBLIOGRAFÍA}

AECA (1997), La Contabilidad de Gestión en los Centros Sanitarios, Asociación Española de Contabilidad y Administración de Empresas, Madrid.

AIBAR, C. (2005), "Seguridad clínica: pequeños pasos y grandes palabras", Revista de calidad asistencial, vol. 20, núm. 4, pp. 183-184.

ANDERSON, R. M. (1986), "Revisiting the behavioral model and the access to Medical Care: Does it matter?", Journal of Health and Social Behaviour, núm. 36, pp. 1-10.

ASHTON, C. M. y otros (1995), "The association between the quality of impaiment care and early readmission", Annals of Internacional Medicine, núm. 122, pp. 415-421.

AVEYARD, P. (1997), "Monitoring the performance of general practices", Journal Evaluation Clinical Practicer, núm.3, pp. 275-281. 
BEGLEY, C. E. y otros (1994), "Avoidable hospitalizations and socio-economics status in Galvenston County, Texas", Journal Community Health, núm. 19, pp. 377-387.

BENBASSAT, J. y TARAGUIN, M. (2000), "Hospital readmissions as a measure of quality of health care: advantages and limitations", Arch Intern Med, núm. 160, pp. 1074-81.

BILLINGS, J. y otros (1996), "Recent finding on preventable hospitalizations", Health Affair, núm. 15, pp. 239-249.

BOHÍGAS, L. (1997), "La acreditación de hospitales: un paso hacia la liberalización del mercado hospitalario español”, en López-Casasnovas, G. y Rodríguez, D. (coords), La regulación de los servicios sanitarios, Civitas, Madrid, pp. 93-128.

BRADFORD, D. F. y otros (1969), "Rising cost of local public services - Some evidence and reflections", National Tax Journal, vol. 22, núm. 2, pp. 185-202.

CAMINAL, J. y CASANOVA, C. (2003), "La evaluación de la atención primaria y las hospitalizaciones por ambulatory care sensitive conditions: Marco conceptual", Atención Primaria, vol. 31, núm. 1, pp. 61-65.

CAPELLA, M. (2000), "Gestión de la calidad en la atención especializada", en Del Llano, J. y otros, (coords), Gestión sanitaria. Innovaciones y desafíos, Masson, Barcelona.

CAREY, T. y otros (1995), "The outcomes and costs of care low back pain among patients seen by primary care practicioners, chiropractors and orthopedic surgeons", The New England Journals of Medicine, vol. 14, núm. 33, pp. 913-917.

CASANOVA, C. y STARFIELD, B. (1995), "Hospitalization of children and access to primary care: a cross-national comparison”, International Journal Health Services, núm. 25, pp. 283-294.

CELLA, D. y otros (2007), "The future of outcomes measurement: Item banking, tailored short-forms, and computerized adaptive assessment", Quality of Life Research, núm. 16 supl. 1, pp. 13-41.

CHARLTON, J. R. H. y otros (1983), "Geographical variation in mortality from conditions amenable to medical intervention in England and Wales”, Lancet, vol. 8326, núm. 1, pp. 691-696.

CLARK, A. (1990), “Are readmissions avoidable?", British Medical Journal, núm. 301, pp. 1136-1138.

CONSEJO DE EUROPA (1998), Recommendation on development and implementation of quality improvement systems (QIS) in health care and explanatory memorandum: 51 .

CREMA, M. y VERBANO, C. (2015), "How to combine lean and safety management in health care processes: A case from Spain”, Safety Science, núm. 79, pp. 63-71. 
CUTLER, D. (2001), "Why has health improved?", Economics 2480, Public economics seminar, Harvard University, Boston.

DE LOS SANTOS ICHASO, M. y GARCÍA-ARMESTO, S. (2012), "Indicadores en atención primaria: la realidad o el deseo", Informe SESPAS, Gaceta Sanitaria, núm. 26(5), pp. 27-35.

D'ANDREAMATTEO, A. y otros (2015), "Lean in healthcare: A comprehensive review", Health Policy, núm. 119, pp. 1197-1209.

DOMÍNGUEZ, J. M. y RUEDA, N. (2005), "Los indicadores de producción pública", Papeles de Economía Española, núm. 105, pp. 77-85.

DONABEDIAN, A. (1966), "Evaluating the quality of medical care", The Milbank Memorial Fund Quarterly, núm. 44, pp. 166-206.

DONABEDIAN, A. (1984), "Volume, quality, and the regionalization of health care services", Medical Care, vol. 22, núm. 2, pp. 95-97.

DONABEDIAN, A. (1988), "The quality of care. How can it be assessed?", Journal of American Medical Association, vol. 12, núm. 260, pp. 1743-1748.

EMERY, M. P. y otros (2005), "Patient-reported outcome and quality of life instruments database (PROQOLID): Frequently asked questions", Health and Quality of Life Outcomes, march, pp. 3-12.

ESPINOZA, P. y otros (2015), "Variabilidad de la distribución en derivaciones a consulta especializada entre profesionales de un centro de salud", Medicina General y de Familia, núm. 4(4), pp. 101-107.

EVANS, R. G. y STODDART, G. L. (1990), "Producing health, consuming health care”, Social Science Medicine, vol. 12, núm. 31, pp. 1347-1363.

FLETCHER, J. y SNEE, H. (1985), "The Need for Output Measurement in the Service Industries: a comment", The Service Industrial Journal, vol. 1, núm. 5, pp. 13-78.

FOGEL, R. (1994), "Economic growth, population theory and physiology: the bearing of long-term processes on the making of economic policy", American Economic Review, núm. 84, pp. 369-395.

FRANKL, SE. y otros (1991), "Preventability of emergent hospital readmission", American Journal of Medicine, núm 90, pp. 667-674.

FUSTÉ, J. y otros (2002), "Hacia la definición de un conjunto mínimo básico de datos de atención primaria", Atención Primaria, núm. 30, pp. 229-235.

GARCÍA ARMESTO, S. y otros (2007), "Health Care Quality Indicators Project 2006 Data Collection Update Report", Documento de trabajo de la OCDE, núm. 29, OCDE, París, http://www.oecd.org/dataoecd/57/22/39447928.pdf, acceso en junio 2016.

GARCÍA-ARMESTO, S. y otros (2010), Spain Health System Review. Health Systems in Transition, 12(4), European Observatory on Health Systems and Policies, Copenhagen, Denmark. 
GARCIA-OLMOS, L. y otros (1995), "Variability in GP, s referral rates in Spain”, Family Practice, vol. 12, núm. 2, pp. 159-162.

GERVÁS, J. y CAMINAL, J. (2007), "Las hospitalizaciones por Ambulatory Care Sensitive Conditions (ACSC) desde el punto de vista del médico de atención primaria”, Revista Española de salud pública, vol. 81, núm. 1, pp. 7-14.

GIL, M. M. (2005), Mortalidad oculta en emergencias sanitarias, Tesis doctoral, Universidad de Granada.

GIMENO, J. A. y otros (2006), "Gestión clínica: Gobierno clínico”, en Oteo, L. A. (coord.) Manuales de Dirección Médica y gestión clínica, Díaz de Santos, Madrid.

GISPERT, R. y otros (2006), "La mortalidad evitable: lista de consenso para la actualización del indicador en España”, Gaceta Sanitaria, núm. 20, pp. 184-193.

GIUFFRIDA, A. y otros (1999), "Measuring quality of care with routine data: avoiding confusion between performance indicators and health outcomes”, BMJ, núm. 319, pp. 94-98.

GOLD, M. (1988), "Common sense on extending the DGR concept to pay form ambulatory care”, Inquiry, vol. 25, núm. 2, pp. 281-289.

GONZÁLEZ, M. y otros (1989), "Los servicios: concepto, clasificación y problemas de medición”, Ekonomiaz: Revista Vasca de Economía, núm. 13-14, pp. 10-19.

GORGEMANS, S. (2013), Eficiencia productiva y dependencia funcional: evidencia en el sector hospitalario español, Tesis doctoral, Universidad de Zaragoza.

GRILICHES, Z. (1992), Output Measurement in the Service Sectors, University of Chicago Press, Chicago, US.

GROSSMAN, H. I. (1979), “Adverse selection, dissembling, and competitive equilibrium", The Bell Journal of Economics, vol. 10, núm. 1, pp. 336-343.

HILL, T. P. (1977), "On goods and services", The Review of Income and Wealth, vol. 23, núm. 2, pp. 315-422.

HOLLAND, R. y otros (1994), "Ductal Carcinoma in-Situation - a Proposal For a New Classification", Seminars in Diagnostic Pathology, núm. 11, pp. $167-180$.

HUTCHINSON, A. y FOWLER, P. (1992), "Outcomemeasuresfor primary health care: what are the research priorities?", British Journal of General Practice, núm. 42, pp. 227-231.

HUTCHINSON, A. y otros (1991), "Case mix measures for ambulatory care”, Journal of Public Health Medicine, vol. 13, núm. 3, pp. 189-197.

IOM (2001), Crossing the Quality Chasm: A New Health System for the 21st Century, Committee on Quality of Health Care in America, National Academies Press, Washington. 
JANOWSKI, R. (1999), "What do hospital admission rates say about primary care?", BMJ, núm. 319, pp. 67-68.

JCAHO (2006), Joint Commission on Accreditation of Healthcare Organizations, http://www.jcaho.org/, acceso en mayo 2016

JUNCOSA, S. y BOLIVAR, B. (1997), "Un sistema de clasificación de pacientes para nuestra atención primaria: los ambulatory care groups (ACGs)", Gaceta Sanitaria, núm. 11, pp. 83-94.

KENDRICK, J. W. (1985), "Measurement of output and productivity in the public sector", en Inman, R. (ed.), Managing the service economy. Prospects and problems, Cambridge University Press, Cambridge, pp. 111-133.

KENT, R. (1985), "Tecnología de servicios y desarrollo económico", Perspectivas económicas, núm. 52, pp. 1-10.

LÓPEZ CASASNOVAS, G. (2003), "El valor de la salud", Humanitas, vol. 1, núm. 3, pp. 183-188.

LÓPEZ CASASNOVAS, G. y RICO, A. (2001), Evaluación de las politicas de servicios sanitarios en el Estado de las Autonomías Vol. II, Fundación BBVA, Bilbao.

LÓPEZ CASASNOVAS, G. y ORTÚN, V. (1998), Economía y Salud, Fundamentos y políticas, Ed. Encuentro, Madrid.

LOPEZ-CASASNOVAS, G. y otros (2005), "Diversity and regional inequalities: assessing the outcomes of the Spanish 'system of health care services", Health Economic, núm. 14, pp. 221-235.

MARMOT, T. y otros (1994), "Inequalities in death. Specific explanations of a general pattern”, Lancet, núm. 1, pp. 1003-1006.

MARTÍNEZ, J. L. (2007), "La gestión de los hospitales", en Villalobos, J. (dir), Gestión sanitaria para los profesionales de la salud, Mc Graw Hill, Madrid.

MATTKE, S. y otros (2006), Health Care Quality Indicators Project Initial Indicators Report, Documento de trabajo de la OCDE, núm. 22, OCDE, París.

MAXWELL, J. R. (1992), "Dimensions of quality revisited: from thought to action", Quality in Health Care, vol. 1, núm. 3, pp. 171-177.

MCCULLOCH, B. y BALL, I. (1992), "Accounting in the context of public sector management reform", Financial Accountability and Management, num. 8, pp. 7-12.

MCKEOWN, T. (1976), The modern rise of population, Academic Press, New York.

MINISTERIO DE SANIDAD Y CONSUMO (1990), Gestión analítica (Hacia la Contabilidad Analítica en los Hospitales), Ministerio de Sanidad y Consumo, Madrid.

MINISTERIO DE SANIDAD Y CONSUMO (2008), Validación de indicadores de calidad utilizados en el contexto internacional: indicadores de seguridad 
de pacientes e indicadores de hospitalización evitable, Ministerio de Sanidad y Consumo, Madrid.

NATIONAL HEALTH SERVICE (1997), A First Class Service-Quality in the new NHS, Department of Health, London, UK.

NEWHOUSE, J. (1993), "An iconoclastic view of Health cost containment", Health Affairs, núm.12 (supl.1), pp. 152-171.

OMS (2000), Informe mundial de la Salud, OMS, Ginebra.

OMS (2006), Quality of Care. Strategic choices in Health Systems, WHO, France.

ORTÚN, V. y otros (1999), Medidas de Producto y eficiencia en Atención Primaria, Documento de Trabajo de la Fundación BBVA, Bilbao.

ORTÚN, V. y GISPER, R. (1988), "Exploración de la mortalidad prematura como guía de política sanitaria e indicador de calidad asistencial", Medicina Clínica, núm. 90, pp. 399-403.

ORTÚN, V. y otros (2004), "El impacto de los servicios sanitarios sobre la salud", https://repositori.upf.edu/bitstream/handle/10230/336/752. pdf?sequence=1, acceso en junio 2016.

PALLOT, J. (1991), "Accounting, Auditing and Accountability", en Boston, J., Martin, J., Pallot, J. y Walsh, P. (eds.), Reshaping the state: New Zealand's Bureaucratic Revolution, Oxford University Press, Auckland, pp. 198-232.

PALMER, R. H. y REILLY, M. C. (1979), "Individual and institutional variables which may serve as indicators of quality of medical care", Medical Care, núm. 17, pp. 693-717.

PEIRÓ, S. (1998), "Medidas de actividad y producto sanitario", en de Llano, J. y otros, (eds.) Gestión sanitaria. Innovaciones y desafíos, Masson, Barcelona, pp. 197-217.

PEIRÓ, S. (2008), "La derivación primaria especializada como problema y como síntoma”, Gestión Clínica y Sanitaria, núm. 10, pp. 84-88

PUIG-JUNOY, J. y DALMAU, E. (2000), "¿Qué sabemos acerca de la eficiencia de las organizaciones sanitarias en España?. Una revisión de la literatura económica", Avances de la Gestión, XX Jornadas de Economía de la Salud, Palma de Mallorca, pp. 151-195.

REALPE, A. X. y otros (2015), "The development of a prototype measure of the co-production of health in routine consultations for people with longterm conditions", Patient Educacion and Counseling, núm. 98, pp. 15401549.

ROGNEHAUGH, R. (1996), The Managed Health Care Dictionary, Gaithersburg, MD, Aspen.

ROMANO, J. y CHOI, A. (2016), "Medida de la eficiencia de la atención primaria en Barcelona incorporando índices de calidad", Gaceta Sanitaria, vol. 30, núm. 5, pp. 359-365. 
ROSS, J. P. y BURKHEAD, J. (1974), Local government; Civil service; Labor productivity, Lexington Books (Lexington, Mass), United States.

ROSSER, R. y WATTS, V. (1978), "The measurement of illness", Journal of the Operational Research Society, vol. 29, núm. 6, pp. 529-540.

RUBILAR, A. y ROSSI, M. T. (2005), "Qué producen los servicios de salud y cómo podemos medirlo?", Revista de la Asociación Médica de Argentina, vol.118, núm. 2, pp. 34-39.

RUBIO, S. (1998), "Economía sanitaria" en Lamata, F. (dir.), Manual de Administración Sanitaria, Díaz de Santos, Madrid.

SALTMAN, R. B. y BANKAUSKAITE, V. (2006), "Conceptualizing decentralization in European health systems: a functional perspective", Health Economics Policy and Law, vol. 1, núm. 2, pp. 127-147.

SALTMAN, R. B. y FIGUERAS, J. (1997), Reforma sanitaria en Europa. Análisis de las estrategias actuales, Ministerio de Sanidad y Consumo y Organización Mundial de la Salud, Madrid.

SERVICIO ANDALUZ DE SALUD (2016), Los ACG en la gestión clínica de atención primaria. Plan de renovación de atención primaria, Consejería de Salud Andaluza. http://www.juntadeandalucia.es/servicioandaluzdesalud/ contenidos/renovacion_ap/que_son_los_ACGs.pdf acceso julio 2016.

SHAW, C. D. y KALO, I. (2002), A background for national quality policies in health systems, World Health Organization, World Health Organization Regional Office for Europe, Copenhagen, Denmark.

SICRAS-MAINAR, A. y otros (2010), "Utilización de los grupos clínicos ajustados en un centro de atención primaria español: estudio retrospectivo de base poblacional", Revista Panamericana de Salud Pública, núm. 27, pp. 49-55.

SICRAS-MAINAR A. y otros, (2007), "Adjusted clinical groups use as a measure of the referrals efficiency from primary care to specialized in Spain”, European Journal Public Health, núm. 17, pp. 657-663.

SIMONATO, L. y otros (1998), "Avoidable mortality in Europe 1955-1994: A plea for prevention", Journal of Epidemiology and Community Health, vol. 52, núm. 10, pp. 624-630.

STARFIELD, B. (1998), Primary care: balancing health needs, services, and technology, Oxford University Press, New York.

STARFIELD B. y otros (1991), "Ambulatory care groups: a categorization of diagnoses for research and management", Health Services Research, núm. 26, pp. 53-74.

THE EUROQOL GROUP. EUROQOL (1990), "A facility for the measurement of health-related quality of life”, Health Policy, núm. 16, pp. 199-207.

THOMAS, J. W. (1996), "Does risk-adjusted readmission rate provide valid information on hospital quality?", Inquiry, núm. 33, pp. 258-270. 
URBINA, O. (2001), Modelización y medida de la eficiencia en atención primaria, Tesis doctoral, Universidad de Zaragoza.

WEINER, J. P. y otros (1991), "Development and application of a populationoriented measure of ambulatory care case-mix", Medical Care, núm. 29, pp. 452-472.

WEISSMAN, J. S. y otros (1999), "Hospital readmissions and quality of care", Medical Care, núm. 37, pp. 490-501.

WESTERLING, R. (2003), "Decreasing gender differences in avoidable mortality in Sweden", Scandinavian Journal of Public Health, vol. 31, núm. 5, pp. 342-349.

WILKINSON, R. (1996), Unhealthy societies, Routledge, Londres.

WORNING, A. M. (1994), Stratégies de réduction des infections nosocomiales. Un modèle pour le développement de la qualité, Etudes de Politique de Santé, OCDE, París.

ZURRO M. A. y JODAR, G. (2011), Atención primaria de salud y atención familiar y comunitaria, Elsevier, Madrid. 